\title{
DUST SPIRALS AND ACOUSTIC NOISE IN THE NUCLEUS OF THE GALAXY NGC $2207^{1}$

\author{
B. G. Elmegreen, ${ }^{2}$ D. M. Elmegreen, ${ }^{3}$ E. Brinks, ${ }^{4}$ C. Yuan,${ }^{5,6}$ M. Kaufman, ${ }^{7}$ \\ M. Klarić, ${ }^{8}$ L. Montenegro, ${ }^{6}$ C. Struck, ${ }^{9}$ And M. Thomasson ${ }^{10}$ \\ Received 1998 May 13; accepted 1998 June 18; published 1998 July 22
}

\begin{abstract}
Observations with the Hubble Space Telescope reveal an irregular network of dust spiral arms in the nuclear region of the interacting disk galaxy NGC 2207. The spirals extend from $\sim 50$ to $\sim 300 \mathrm{pc}$ in galactocentric radius, with a projected width of $\sim 20 \mathrm{pc}$. Radiative transfer calculations determine the gas properties of the spirals and the inner disk and imply a factor of $\sim 4$ local gas compression in the spirals. The gas is not strongly self-gravitating, nor is there a nuclear bar, so the spirals could not have formed by the usual mechanisms applied to main galaxy disks. Instead, they may result from acoustic instabilities that amplify at small galactic radii. Such instabilities may promote gas accretion into the nucleus.
\end{abstract}

Subject headings: hydrodynamics — instabilities — ISM: structure — galaxies: individual (NGC 2207) — galaxies: nuclei

\section{INTRODUCTION}

Recent high-resolution observations of the nuclear regions of some spiral galaxies show irregular spiral patterns in dust and gas that are not continuations of the main spiral arms or driven by obvious bars. Examples in the recent literature are NGC 278 (Phillips et al. 1996), M81 (Devereux, Ford, \& Jacoby 1997), M87 (Ford et al. 1994; Dopita et al. 1997), NGC 4414 (Thornley \& Mundy 1997), NGC 5188, and NGC 6810 (Carollo, Stiavelli, \& Mack 1998).

This Letter reports similar spirals within the inner $500 \mathrm{pc}$ of NGC 2207 that were found in the course of a larger program to study this galaxy and its interacting neighbor, IC 2163, with the Hubble Space Telescope (HST). NGC 2207 and IC 2163 were previously observed from the ground at optical and radio $(21 \mathrm{~cm})$ wavelengths (Elmegreen et al. 1995a; hereafter, Paper I) and modeled by computer simulations to reproduce the peculiar morphologies, internal velocities, and orbital motions of the main galaxy disks (Elmegreen et al. 1995b).

The nuclear spirals in NGC 2207 are too small to observe by any present-day instrument other than $H S T$, so we derive their properties and those of the nuclear disk using only the HST data, combined with radiative transfer techniques. The disk is found to be too weakly self-gravitating for the spirals to be density waves. We investigate other possible origins for the observed structure.

\footnotetext{
${ }^{1}$ Based on observations with the NASA/ESA Hubble Space Telescope, obtained at the Space Telescope Science Institute, which is operated by the Association of Universities for Research in Astronomy, Inc. under NASA contract NAS5-26555.

${ }^{2}$ IBM Research Division, T. J. Watson Research Center, P.O. Box 218, Yorktown Heights, NY 10598; bge@watson.ibm.com.

${ }^{3}$ Department of Astronomy, Vassar College, Poughkeepsie, NY 12604.

${ }^{4}$ Departmento de Astronomía, Universidad de Guanajuato, Apartado Postal 144, Guanajuato, Gto. 36000, México.

${ }^{5}$ Institute of Astronomy and Astrophysics, Academia Sinica, Nankang, Taipei, Taiwan, ROC.

${ }^{6}$ City College of New York, 138th Street on Convent Avenue, New York, NY 10031.

${ }^{7}$ Department of Physics and Department of Astronomy, Ohio State University, 174 West 18th Avenue, Columbus, $\mathrm{OH} 43210$.

${ }^{8}$ Midlands Technical College, Columbia, SC 29202.

${ }^{9}$ Department of Physics and Astronomy, Iowa State University, Ames, IA 50010.

${ }^{10}$ Onsala Space Observatory, S-439 92 Onsala, Sweden.
}

\section{OBSERVATIONS}

In 1996 May, we observed the nuclear region of NGC 2207 with the refurbished Planetary Camera of the HST at 0.0455 resolution, using filters at four passbands: $U, B, V$, and $I$. A color image made from the $V$ and $I$ bands of a region around the nucleus is shown in Figure 1 (left). The inner $\mathrm{H}$ I disk (Paper I) is inclined by $35^{\circ}$ and has a major axis position angle of $\sim 140^{\circ}$; approximately the same inclination applies to the nuclear disk seen here (from the ellipticity of the intensity distribution), but the position angle is $\sim 189^{\circ}$. (Note the arrow in the bottom left-hand corner of the HST image, which indicates north.) The image on the right in Figure 1 is an unsharpmasked version of the $V$ image, which highlights the dust structure. The angular scale of the figure is 160 pixels $=7 " .28$ on a vertical side, which is $1.24 \mathrm{kpc}$ for a distance of $35 \mathrm{Mpc}$ (Paper I).

Several dark dust streamers are evident in the figure. They are peculiar in several respects: (1) they have no accompanying stellar spiral arms or star formation knots (confirmed by $U$ and $B$ images, which are not shown here because their signal-tonoise ratios are lower than for the $V$ and $I$ images), as do dustlanes in the main disks of spiral galaxies, (2) they have much larger pitch angles $\left(\sim 50^{\circ}\right)$ than main-disk spirals (which have pitch angles of $\sim 15^{\circ}$ ), (3) there are many of them, particularly in the outer parts, forming a dark pinwheel with some intersections, not just a regular two-arm spiral as in granddesign galaxy disks, and (4) they are very short, with the shortest only several tens of parsecs long and the longest several hundred parsecs.

The nucleus has an exponentially varying light profile near the center, as shown at the top of Figure 2. The curves are intensity scans across the nucleus in $V$ and $I$ bands, averaged over a scan width of 5 pixels, along a strip parallel to the two arrows shown at the top of the left-hand image in Figure 1. There is no power-law cusp (even on single pixel-wide scans) and hence no obvious black hole that might be revealed by such a cusp (Lauer et al. 1992). This is consistent with the lack of a nuclear radio continuum source (Condon 1983; Vila et al. 1990) from an active nucleus. Neither is there a nuclear starburst (Paper I). The exponential scale length in $V$ and $I$ bands within 0.5 from the center is $\sim 0.47$, or 80 pc deprojected. Just beyond this distance, the deprojected scale lengths change to $360 \mathrm{pc}$ in $V$ and $270 \mathrm{pc}$ in $I$. 


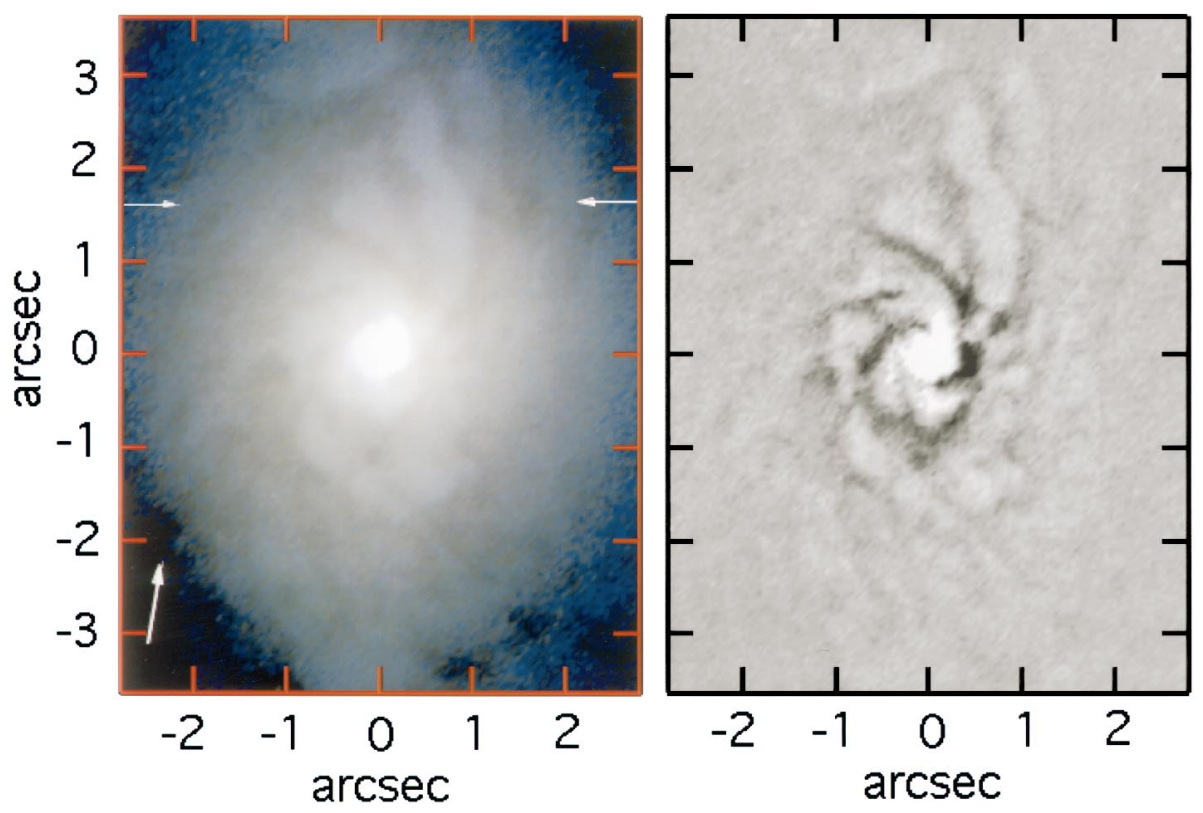

FIG. 1.-Left: HST image of the nuclear region of NGC 2207 in color $r g b$ format, with $r$ taken to be the $I$-band image, $b$ the $V$-band image, and $g$ the average of these two images. Arrows at the top indicate the strip used to fit the radiative transfer model. North is indicated by the arrow in the bottom left-hand corner. Right: Unsharp-masked image of the $V$-band showing intricate dust structure as dark bands. The unsharp mask was made by subtracting the convolution of the $V$ image with a Gaussian of 0.5 FWHM from the original $V$ image.

\section{RADIATIVE TRANSFER MODEL}

In order to estimate gas densities, the dust spiral was studied using a radiative transfer model (Block, Elmegreen, \& Wainscoat 1996) that was fit to an approximately east-west scan positioned 1".638 = $278 \mathrm{pc}$ north of the nucleus (see arrows, Fig. 1). The $V$ - and $I$-band intensity profiles along the scan are shown at the bottom of Figure 2, along with the model fits
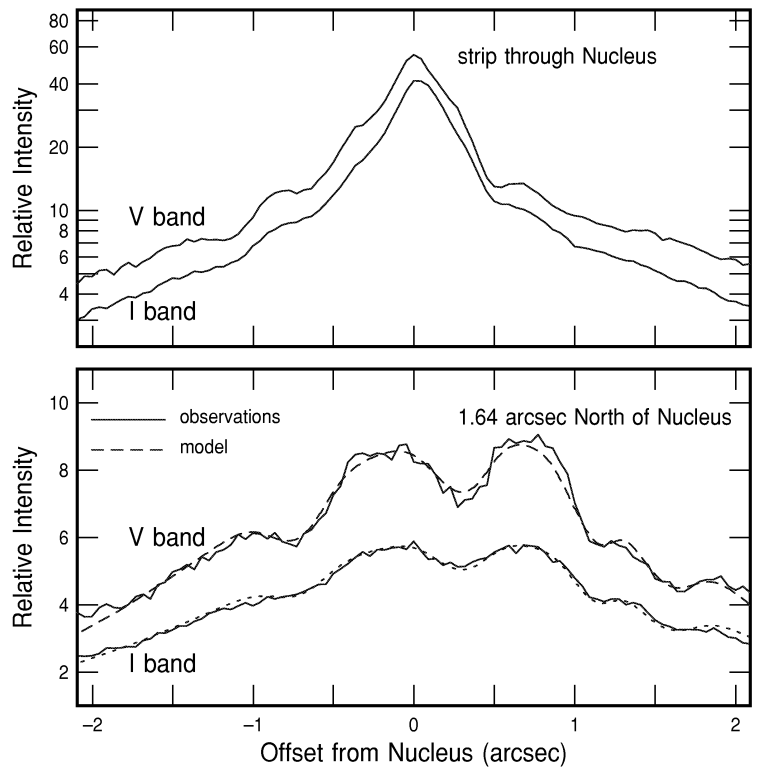

FIG. 2.-Top: Intensity profiles in $V$ and $I$ bands along a strip 5 pixels (= 0 "228) wide through the nucleus, parallel to the strip used for the radiative transfer model, shown by the arrows in Fig. 1. Bottom: Intensity profiles in $V$ and $I$ bands through a strip north of the nucleus and radiative transfer fits (dashed lines) to these profiles. (dashed lines). The model considers an inclined disk with properties that are typical for galaxies: exponential radial profiles with the same scale lengths for the stars, gas, and dust; Gaussian profiles perpendicular to the disk with a smaller scale height $h$ for the gas and dust mixture than the stars (for which $h=$ $100 \mathrm{pc}$ is assumed); and the same scale heights for the dark features as for the regions between them. Note that the value of the stellar scale height does not matter much as long as it is several times larger than the gaseous scale height. Four dust features with Gaussian profiles parallel to the disk cause the absorption dips seen in the scans. The fit was made to the $I$ band because extinction is weakest there; only the ratio of extinction to gas column density was varied between $V$ and $I$ bands, as appropriate for these two wavelengths. Scattering is included in the models but is found to be relatively unimportant.

Some aspects of the model are uniquely specified by the data. The observed widths of the dust features have to match the projected dimensions in the models, and then the midplane densities in the dust features are determined uniquely by the line-of-sight opacities of the absorption dips. The intrinsic disk scale length in the model is also determined by the observations, but only approximately because internal absorption flattens the disk profile (Byun, Freeman, \& Kylafis 1994). As a result, the model gives the internal extinction, the gas surface density between the dust lanes, and the intrinsic disk scale length, but each are uncertain by a factor of $\sim 2$.

The radiative transfer fit in Figure 2 has a $V$-band extinction in the midplane at the position of the strip equal to $\sim 9$ optical depths per kiloparsec between the dustlanes. This corresponds to an average hydrogen density of $\sim 6 \mathrm{~cm}^{-3}$ with the conventional dust-to-gas conversion factor (Bohlin, Savage, \& Drake 1978) and the usual assumption that gas and dust are well mixed. The perpendicular scale height of the inner disk is $\sim 35$ $\mathrm{pc}$, and the intrinsic exponential scale lengths of the $\mathrm{V}$ - and $I$ band emissivities are 170 and $220 \mathrm{pc}$ (these are less than the observed scale lengths because absorption in the disk flattens 
the apparent intensity profile). The hydrogen densities in the four dustlanes, in order from east to west (left to right in Fig. 2 ), are $11,17,45$, and $16 \mathrm{~cm}^{-3}$, and their in-plane dimensions are $\sim 20,20,5$, and $25 \mathrm{pc}$. The average ratio of the density in a dustlane to the density between the dustlanes is $\sim 4$. Most of the projected extents of these dustlanes are from the perpendicular disk scale height. The products of the line-of-sight dustlane thicknesses and the midplane densities give effective lineof-sight $V$-band extinctions through the dust features of $\sim 1.2$, $1.8,1.2$, and 2.1 optical depths, respectively. The total extinction between the dustlanes, perpendicular to the disk, is the product of twice the disk scale height and the opacity, or 0.6 optical depths in $V$ band.

The radiative transfer solution is the only way to estimate the gas density at high resolution; the radio observations are at much lower resolution. The radiative transfer suggests that the average hydrogen column density between the dustlanes at this radius, measured perpendicular to the disk, is $\sim 1.3 \times$ $10^{21} \mathrm{~cm}^{-2}$, which corresponds to a mass column density of 14 $M_{\odot} \mathrm{pc}^{-2}$ including helium. This is larger than the inclinationcorrected VLA ${ }^{11}$ measurement (Paper I) of $\sim 3 M_{\odot} \mathrm{pc}^{-2}$ for $\mathrm{H}$, including helium, in a $13^{\prime \prime} .5 \times 12^{\prime \prime}$ beam centered on the nucleus. There are no direct $\mathrm{CO}$ observations of the nucleus, but there are two $\mathrm{CO}$ detections made by one of us (M. T.) with a 43" beam at the Swedish-ESO Submillimetre Telescope (La Silla, Chile), centered one-fourth-beam east and west of the nucleus; both detections include the nucleus in the beam pattern. The integrated $\mathrm{CO}$ emissions are each $6.9 \mathrm{~K} \mathrm{~km} \mathrm{~s}^{-1}$ on the main beam temperature scale, so the inclination-corrected mass column density for molecular hydrogen plus helium is $29 M_{\odot} \mathrm{pc}^{-2}$, assuming the standard conversion factor for CO luminosity to molecular mass (Strong et al. 1988). All of these observations suggest that the nuclear disk has a relatively low gas column density, which is consistent with the lack of a starburst in the inner region.

\section{POSSIBLE ORIGINS FOR THE NUCLEAR SPIRALS}

What is the origin of the dust spirals in the nuclear disk of NGC 2207? Spirals like these are not typically present in galaxies with strong nuclear or inner ring starbursts, where the dust features are accompanied by star formation (e.g., NGC 6946: Engelbracht et al. 1996), nor are they as symmetric as dust features in bars (e.g., NGC 4321: Sakamoto et al. 1995; Knapen et al. 1995). The nuclear dust spirals are also not likely to be related to the main spiral structures because the nuclear spirals lie inside the inner Lindblad resonance for the outer spirals (Elmegreen, Elmegreen, \& Montenegro 1992) and inside a $Q$ barrier that might come from a bulge. Both of these features shield the nuclear region from incoming spiral waves (Bertin et al. 1989). The position inside the inner resonance also precludes any resonance excitation that might come from the outer spirals (Sellwood \& Lin 1989; Sellwood \& Kahn 1991).

This leads us to suspect that the spirals are formed and dispersed continuously in the nuclear disk. They could be strong compressions or shock fronts caused by turbulent motions that are driven by gas inflow (Struck 1997) or other irregularities in the nuclear disk, or they could result from local instabilities.

\footnotetext{
${ }^{11}$ The National Radio Astronomy Observatory (NRAO) is operated by Associated Universities, Inc., under cooperative agreement with the National Science Foundation.
}

The magnetically driven instability discussed by Balbus \& Hawley (1991) is a reasonable choice, discussed already in this context by Dopita et al. (1997). However, it might not be appropriate for low-density nuclear disks like NGC 2207 because it requires high angular shear. The disk in M87 studied by Dopita et al. surrounds a black hole and probably has the required shear, but the situation in NGC 2207 is not so clear. We would prefer an explanation for the spirals in NGC 2207 that works with little shear.

Another well-known instability produces multiple-arm spirals in the main disks of galaxies and is driven by the gravity of the stars and gas (Lin \& Shu 1964; Toomre 1981). This instability becomes strong when a dimensionless parameter $Q=\kappa a /(\pi G \sigma)$ has a value of around 2 or less (Toomre 1981), and it continues to work with small angular shear if $Q \sim 1$. Here $\kappa$ is the frequency of weak radial oscillations for slightly displaced material, $a$ is the rms velocity dispersion, $G$ is the gravitational constant, and $\sigma$ is the gas mass column density of the disk. We do not observe $a$ and $\kappa$ in the nuclear region directly because the angular scale is too small, but we can estimate them from the radiative transfer solution. We consider solid-body rotation at angular rate $\Omega$, for which $\kappa=2 \Omega$, and treat first a non-self-gravitating disk, where the scale height is $h=a / \Omega$. These combine to give $Q=2 a^{2} /(\pi G \sigma h)$. The radiative transfer solution gives $\sigma \sim 14 M_{\odot} \mathrm{pc}^{-2}$ and $h=35 \mathrm{pc}$ in the vicinity of the strip. Thus $Q \sim 0.3 a^{2}$ with $a$ in kilometers per second. The VLA data (Paper I) suggest that the entire disk, including the inner portion, has a relatively large gaseous velocity dispersion, possibly in excess of $40 \mathrm{~km} \mathrm{~s}^{-1}$. If $a\left(\mathrm{~km} \mathrm{~s}^{-1}\right)>10$, which is likely, then $Q$ is much larger than 2, consistent with our use of $h \sim a / \Omega$. We note that this estimate for $\Omega \sim a / h \sim 0.28 \mathrm{~km} \mathrm{~s}^{-1} \mathrm{pc}^{-1}$ is also consistent with groundbased optical observations (Rubin \& Ford 1983) at 1" resolution. This implies that even the usual expression for $Q$, which is valid for a self-gravitating disk as well, gives a large value. Setting $\Omega>0.1 \mathrm{~km} \mathrm{~s}^{-1} \mathrm{pc}^{-1}$ from Rubin et al., we get $Q=$ $\kappa a /(\pi G \sigma)>1.1 a$ for $a$ in kilometers per second. This is again much larger than 2 for reasonable velocity dispersion $a$. Because of the large $Q$, the self-gravitational instability that drives spirals in the main disks of galaxies cannot operate in the nuclear disk of NGC 2207.

If self-gravity cannot generate the spirals, then another local source must be present. An intriguing possibility is the natural tendency for sound waves to amplify at small galactocentric radii (Montenegro 1998; Montenegro, Yuan, \& Elmegreen 1998). Consider a disk in rigid rotation with constant surface density (exponential variations in $\sigma$ and $a$ and radial variations in $\Omega$ do not affect the results significantly). The governing equation for a wavelike perturbation in column density, $\sigma_{1} \sim$ $h_{1} e^{i(\omega t-m \theta)}$, is

$$
\frac{d^{2} h_{1}}{d r^{2}}+\frac{1}{r} \frac{d h_{1}}{d r}+\left[\frac{\kappa^{2}}{a^{2}}\left(\nu^{2}-1\right)-\frac{m^{2}}{r^{2}}\right] h_{1}=0
$$

Here $\nu \equiv(\omega-m \Omega) / \kappa, m$ is the azimuthal wavenumber, $r$ is the galactocentric radius, and $\Omega, \kappa$, and $a$ were defined above. When

$$
\frac{\kappa^{2}}{a^{2}}\left(\nu^{2}-1\right) \equiv \alpha^{2}>0
$$

the solutions are Bessel functions $J_{m}(\alpha r)$ and $Y_{m}(\alpha r) ; Y_{m}$ diverges at small $\alpha r$. For $\alpha r \sim \kappa r / a$ large, $J_{m}$ and $Y_{m}$ combine to 
give outgoing and incoming sonic waves. In NGC 2207, there is little activity in the nucleus; the waves generated locally are probably moving in both directions. Those that propagate outward become weaker by geometric effects (amplitude proportional to $r^{-1 / 2}$ ) and viscous damping. Those that propagate inward amplify as $Y$ diverges; then they dissipate by shocks when $r$ approaches the center near $r=m / \alpha \sim m a / \kappa$. At this radius, the separation between waves equals the minimum possible distance, which is the radius of epicyclic motion at the sound speed. Thus, waves with high $m$ occupy mostly the outer regions of the nuclear disk. This result is in agreement with the observations in Figure 1: there are many more dust features in an annulus at large radius than at small radius.

The waves also propagate in the azimuthal direction with angular speed $\omega / m$, as long as $\nu^{2}-1>0$. Waves with different $m$ therefore interact to form complex structures and intersections, as seen in NGC 2207. The waves may also become sheared by slight differential rotation to form open trailing spirals, although shear is not required to make the gas unstable.

The above solutions assume that the frequency $\omega$ is real and that the radial wavenumber, $k \equiv-i d$ (phase) $/ d r$, is complex. We can assume alternatively that $k$ is real and $\omega$ is complex, in which case the waves, fixed in space, grow in time. The result (Montenegro et al. 1998) is a growth time for the waves equal to the propagation time, $r / a$, and because $r / h \sim r \Omega / a \sim$ 6 in the part of NGC 2207 considered here, this is also about the orbit time. After this time, the waves shock and dissipate, as in the solution $Y$ above.

These properties of sonic noise at small galactocentric radii should be appropriate for a nuclear galactic disk that is not dominated by a strong bar, a black hole, or a starburst. They agree well with the characteristics of the dust features observed at the center of NGC 2207. Acoustic spirals have not been considered for galaxies before because the main disks typically have sound speeds much less than orbit speeds, and they also have much stronger self-gravity. Nevertheless, acoustic spirals may be an important agent for driving gas accretion in some nuclei.

The accretion rate from acoustic spirals may be estimated from the energy dissipation rate. If the waves become mild shocks, then the energy flux into each shock is $\sim 1.5 \rho a^{3}$, and the total shock dissipation rate is $\sim 1.5 \rho a^{3} \times 2 h \times m L$ for number of arms $m$ and arm length $L$. Dividing this by the orbital energy available, which is $\sim 0.5 \rho v^{2} \times 2 h \times \pi r^{2}$ for orbit speed $v$, the loss rate becomes several times $a^{3} \mathrm{~m} /\left(v^{2} r\right)$, considering that $L \sim r$. Now we can use $r \sim m a / \kappa$ to describe the radius of wave shocking, from the Bessel function solution above, and then the overall evolution rate is $\sim \kappa(a / v)^{2}$. The mass accretion time is therefore comparable to the orbit time multiplied by the square of the ratio of the orbit speed to the sound speed. In nuclear regions like this, the orbit speed may be only a few times the sound speed, and then the gas accretion time can be relatively short. Presumably there is self-regulation, however, because persistent shocking in acoustic waves might heat the gas to the point at which shocks no longer appear. Then the accretion process would slow down until the gas cools again. The overall impact of acoustic waves on accretion is therefore difficult to assess.

\section{SUMMARY}

NGC 2207 contains peculiar dust spiral arms in its nuclear disk that are not obviously related to star formation, bar structure, or gravity-driven spiral density waves. They could be regions of forced compression that are stirred, for example, by inflow, but they could also be amplified acoustic waves, which tend to drive spiral structure at small relative radii (i.e., $r \kappa / a$ not much larger than 1). The resulting spiral arms steepen and shock in an orbit time, which is only $\sim 2 \times 10^{7} \mathrm{yr}$ in the region studied, and this shocking ultimately causes the gas to lose angular momentum and energy. Then it can accrete to the nucleus.

Acoustic spirals may be the primary agent for gas accretion in the most quiescent nuclear disks, i.e., those with weak selfgravity and bar forcing, limited access to outer disk activity, and little shear. It may also be an important supplement to other accretion processes, such as bar-driven shocks and the Balbus \& Hawley (1991) instability, in more active regions. A more quantitative assessment of its role in general nuclear environments is difficult to make at the present time.

Support for this work was provided by NASA through grant number GO-06483-95A from the Space Telescope Science Institute, which is operated by the Association of Universities for Research in Astronomy, Inc., under NASA contract NAS526555. Figure 1 was made using the IBM Data Explorer software, with the help of Tom Jackman.

\section{REFERENCES}

Balbus, S. A., \& Hawley, J. F. 1991, ApJ, 376, 214

Bertin, G., Lin, C. C., Lowe, S. A., \& Thurstans, R. P. 1989, ApJ, 338, 78

Block, D. L., Elmegreen, B. G., \& Wainscoat, R. J. 1996, Nature, 381, 674

Bohlin, R. C., Savage, B. D., \& Drake, J. F. 1978, ApJ, 224, 132

Byun, Y. I., Freeman, K. C., \& Kylafis, N. D. 1994, ApJ, 432, 114

Carollo, C. M., Stiavelli, M., \& Mack, J. 1998, AJ, in press

Condon, J. J. 1983, ApJS, 53, 459

Devereux, N., Ford, H., \& Jacoby, G. 1997, ApJ, 481, L71

Dopita, M. A., Koratkar, A. P., Allen, M. G., Tsvetanov, Z. I., Ford, H. C., Bicknell, G. V., \& Sutherland, R. S. 1997, ApJ, 490, 202

Elmegreen, B. G., Elmegreen, D. M., \& Montenegro, L. 1992, ApJS, 79, 37

Elmegreen, D. M., Kaufman, M., Brinks, E., Elmegreen, B. G., \& Sundin, M. 1995a, ApJ, 453, 100

Elmegreen, B. G., Sundin, M., Kaufman, M., Brinks, E., \& Elmegreen, D. M. 1995b, ApJ, 453, 139

Engelbracht, C. W., Rieke, M. J., Rieke, G. H., \& Latter, W. B. 1996, ApJ, 467,227

Ford, H. C., et al. 1994, ApJ, 435, L27
Knapen, J. H., Beckman, J. E., Heller, C. H., Shlosman, I., \& de Jong, R. S. 1995, ApJ, 454, 623

Lauer, T. R., et al. 1992, AJ, 103, 703

Lin, C. C., \& Shu, F. H. 1964, ApJ, 140, 646

Montenegro, L. 1998, Ph.D. thesis, City College of New York

Montenegro, L., Yuan, C., \& Elmegreen, B. G. 1998, ApJ, submitted

Phillips, A. C., Illingworth, G. D., MacKenty, J. W., \& Franx, M. 1996, AJ, 111,1566

Rubin, V. C., \& Ford, W. K., Jr. 1983, ApJ, 271, 556

Sakamoto, K., Okumura, S., Minezaki, T., Kobayashi, Y., \& Wada, K. 1995, AJ, 110, 2075

Sellwood, J. A., \& Kahn, F. D. 1991, MNRAS, 250, 278

Sellwood, J. A., \& Lin, D. N. C. 1989, MNRAS, 240, 991

Strong, A. W., et al. 1988, A\&A, 207, 1

Struck, C. 1997, ApJS, 113, 269

Thornley, M. D., \& Mundy, L. G. 1997, ApJ, 490, 682

Toomre, A. 1981, in The Structure and Evolution of Normal Galaxies, ed. S. M. Fall \& D. Lynden-Bell (Cambridge: Cambridge Univ. Press), 111

Vila, M. B., Pedlar, A., Davies, R. D., Hummel, E., \& Axon, D. J. 1990, MNRAS, 242, 379 